\title{
New Semi-Quantification Approach for Dopamine Transporter Scan: Quantification of Accumulation by Examining the Approximate Image
}

Yoshinori lto

Nagoya University Graduate School of Medicine

Naotoshi Fujita

Nagoya University Graduate School of Medicine

Kazuhiro Hara

Nagoya University Graduate School of Medicine

Tomohiro Tada

Nagoya University Graduate School of Medicine

Shinji Abe

Nagoya university Hospital

Masahisa Katsuno

Nagoya Univerisity Graduate School of Medicine

Shinji Naganawa

Nagoya University Graduate School of Medicine

Katsuhiko Kato ( $\nabla$ katokt@med.nagoya-u.ac.jp )

Nagoya University Graduate School of Medicine https://orcid.org/0000-0002-2083-9319

Original research

Keywords: FP-CIT, Specific binding ratio, Quantification, Dopamine transporter, SPECT

Posted Date: December 22nd, 2020

DOI: https://doi.org/10.21203/rs.3.rs-131048/v1

License: (1) (1) This work is licensed under a Creative Commons Attribution 4.0 International License.

Read Full License 
1 Title

2 New Semi-quantification Approach for Dopamine Transporter Scan: Quantification of

3 Accumulation by Examining the Approximate Image

4

5 Authors

6 Yoshinori (to'), Naotoshi Fujita1), 2), Kazuhiro Hara ${ }^{3)}$, Tomohiro Tada1), Shinji Abe ${ }^{2)}$, Masahisa

7 Katsuno ${ }^{3)}$, Shinji Naganawa ${ }^{4)}$, Katsuhiko Kato ${ }^{5)}$

8

9 Institutional addresses

10 1) Department of Radiological and Medical Laboratory Sciences, Nagoya University Graduate

11 School of Medicine, 1-1-20 Daiko-Minami, Higashi-ku, Nagoya 461-8673, Japan

12 2) Department of Radiological Technology, Nagoya University Hospital, 65 Tsurumai-cho,

13 Showa-ku, Nagoya 466-8560, Japan

14 3) Department of Neurology, Nagoya University Graduate School of Medicine, 65 Tsurumai-

15 cho, Showa-ku, Nagoya 466-8550, Japan

16 4) Department of Radiology, Nagoya University Graduate School of Medicine, 65 Tsurumai17 cho, Showa-ku, Nagoya, 466-8550, Japan

18 5) Functional Medical Imaging, Biomedical Imaging Sciences, Division of Advanced 19 Information Health Sciences, Department of Integrated Health Sciences, Nagoya University 20 Graduate School of Medicine, 1-1-20 Daiko-Minami, Higashi-ku, Nagoya 461-8673, Japan

\section{Corresponding author}

Katsuhiko Kato

4) Functional Medical Imaging, Biomedical Imaging Sciences, Division of Advanced Information Health Sciences, Department of Integrated Health Sciences, Nagoya University Graduate School of Medicine, 1-1-20 Daiko-Minami, Higashi-ku, Nagoya 461-8673, Japan

[Phone number] +81-52-719-1590

[Fax number] +81-52-719-1589

[Email address] katokt@med.nagoya-u.ac.jp 


\section{Abstract}

\section{Background}

33 Semi-quantitative analysis is used to evaluate the degree of tracer binding to the striatum in 34 dopamine transporter (DaT) single-photon emission computed tomography (SPECT). In DaT SPECT, it is difficult to evaluate the accurate tracer accumulation due to the partial volume effect (PVE). In this study, we propose a novel semi-quantitative approach for measuring the amount of accumulation by examining the approximate image. Using the striatal phantom, we verified the validity of a newly proposed method that can accurately evaluate the tracer accumulations in the caudate and putamen, separately.

41 The left and right caudate/putamen regions, and the whole brain region as the background (BG) region were identified in computed tomography $(\mathrm{CT})$ images obtained by SPECT/CT imaging, and the positional information of each region was obtained. The SPECT-like images were generated by assigning assumed accumulation amounts to the left and right caudate/putamen and BG regions based on the positional information. By changing the assumed accumulation amounts assigned to each region, the SPECT-like image, which was approximated to the image obtained by the SPECT imaging, was examined. The accumulation amounts assumed, when the generated SPECT-like image approximated the most to the image actually obtained by the SPECT imaging, were determined as the accumulation amounts in each region. We evaluated the correlation between the count density calculated by the proposed method and the actual count density of ${ }^{123}$ solution filled into the striatal phantom, and verified the validity of the proposed method. In addition, the specific binding ratio (SBR) and caudate-putamen ratio (CPR) computed by the proposed method were compared with the theoretical SBR and CPR calculated by the count density of the ${ }^{123}$ I solution filled into the striatal phantom.

Results

The count density calculated by the proposed method and the count density of ${ }^{123}$ | solution filled in the striatal phantom had an extremely strong positive correlation $(r=0.997, p<0.001)$. The SBRs computed by the proposed method were overestimated. However, the obtained CPRs were very similar to the theoretical CPRs.

\section{Conclusions}


62 The proposed method was able to compute the accurate accumulation amounts in the 63 caudate and putamen, considering the PVE.

64

\section{Keywords}

66 FP-CIT, Specific binding ratio, Quantification, Dopamine transporter, SPECT

67

68

69

70

71

72

73

74

75

76

77

78

79

80

81

82

83

84

85

86

87

88

89

90

91

92 


\section{Background}

Dopamine transporter (DaT) scintigraphy with ${ }^{123} \mathrm{I}-\mathrm{N}-\omega$-fluoropropyl-2 $\beta$-carboxymethoxy-3 $\beta$ -(4-iodophenyl)nortropane (123/-FP-CIT) is useful in the diagnosis of Parkinson's disease (PD) and dementia with Lewy bodies (DLB) [1-3]. The specific binding ratio (SBR) is widely used to evaluate the degree of tracer binding to the striatum. However, with the spatial resolution of single-photon emission computed tomography (SPECT), it is difficult to accurately evaluate the accumulation amount of the tracer owing to small structures such as the striatum, which are affected by the partial volume effect (PVE) [4]. The Southampton method [5], which is widely used in Japan, can calculate the SBR considering the PVE by using a large volume of interest (VOI) - including the entire striatum. However, this method cannot calculate the SBR for the caudate and putamen, separately. It has been reported that in PD, the ventrolateral region of the substantia nigra is selectively impaired, resulting in severe dopamine loss in the dorsal putamen compared to the caudate [6]. Therefore, in PD, accumulation of ${ }^{123}$ I-FP-CIT is especially reduced in the dorsal putamen. Moreover, in DLB, accumulation of ${ }^{123} \mathrm{I}-\mathrm{FP}-\mathrm{CIT}$ is uniformly reduced in the caudate and putamen $[7,8]$. Furthermore, it has been reported that the caudate is related to the cognitive function and the putamen is related to the motor function, and it is suggested that the accumulation amount of ${ }^{123}$ I-FP-CIT is also related to this $[6,7]$. Based on this information, evaluation of the accurate accumulation amount in the caudate and putamen is anticipated to lead to improved diagnostic accuracy for PD. Furthermore, it is considered that the measurements of the accumulation in the caudate and putamen, separately, may lead to evaluation of their respective functions, that is, the caudate and cognitive function, and putamen and motor function. In this study, we propose a novel semi-quantitative approach of measuring the accumulation amount by examination of the approximate image. The study aimed to verify, using the striatal phantom, a newly proposed method that can accurately evaluate the accumulation amounts in the caudate and putamen.

\section{Methods}

\section{2-1. SPECT imaging}

SPECT data were acquired using Symbia T (Siemens, Erlangen, Germany), equipped with a low-medium energy general purpose (LMEGP) collimator. Ninety projections over $360^{\circ}$ orbit 
with two detectors were acquired on a $128 \times 128$ matrix with a zoom of 1.45 , giving a pixel size of $3.3 \mathrm{~mm}$ and acquisition time of $28 \mathrm{~min}$. The main energy window was $159 \mathrm{keV} \pm 10 \%$ and two subwindows were set at $8 \%$ at both ends of the main window. Images were reconstructed using a three-dimensional ordered subset expectation maximization method (3D-OSEM) (iteration, 6; subset, 8) and Gaussian filter full width at half maximum (FWHM) 6 $\mathrm{mm}$ with attenuation correction (AC) by computed tomography (CT) and scatter correction (SC) using the triple energy window (TEW) method.

\section{2-2. Phantom data}

The striatal phantom DaT1308 (NMP Business Support Co., Ltd., Hyogo, Japan) was used. We filled ${ }^{123}$ I solution into the right caudate and left caudate regions of the striatal phantom at a scheduled radioactivity concentration ratio of 1.80:1.00, and water into the right and left putamen regions, and the whole brain region which considered as background (BG). A phantom created by this condition of radioactivity concentration ratio was defined as "Phantom1." Then, we filled ${ }^{123}$ I solution into the right caudate, right putamen, left caudate, left putamen, and BG regions of the striatal phantom at a scheduled radioactivity concentration ratio of 9.00:9.00:5.00:4.00:1.00. A phantom created by this condition was defined as "Phantom2." We measured the actual count densities of the ${ }^{123}$ I solution filled into "Phantom1" and "Phantom2" by an auto-well counter (ARC-7001, Hitachi Aloka Medical, Ltd., Tokyo, Japan) and computed the actual count density ratio. The data of the created "Phantom1" and "Phantom2" are shown in Table 1.

\section{2-3. Calculation process by the proposed method}

The definitions of the terms used in the calculation process of the proposed method are shown in Table 2. Moreover, an overview of the calculation process of the proposed method is shown in Fig. 1.

2-3-1. Process No. 1 of the proposed method: Setting volume of interests (VOIs) and creating mask images (Fig. 2)

Using the PMOD software version 3.903 (PMOD Technologies LCC, Zurich, Switzerland), we manually established the VOIs for the left and right caudate/putamen, and BG regions along 
the morphology in the CT images obtained by SPECT/CT. These VOIs on CT images were applied to the SPECT images. The mask images, which extracted the positional information of the left and right caudate /putamen, and the BG regions on SPECT images, were created according to the VOIs on the SPECT images, thereby obtaining the anatomical positions for each region.

\section{2-3-2. Process No. 2 of the proposed method: Generating "assigned image" and "blurred} image" (Fig. 3)

Process No. 1 was processed using PMOD software, and Process No. 2 or later was processed on our own program built using the programing language Python version 3.7.3.

Based on the positional information of the left and right caudate/putamen BG regions obtained by Process No. 1, five values of $0,0.5,1.0,1.5$, and 2.0, as assumed accumulation amounts assigned to each region. Since five values (i.e., $0,0.5,1.0,1.5,2.0$ ) were assigned to the five regions (the left and right caudate/putamen and BG regions), five to the fifth power (3125) images with no blur (we defined these images as "assigned images") were generated. Three-dimensional (3D) Gaussian filter equivalent to SPECT spatial resolution in clinical conditions (FWHM: $x=11.52 \mathrm{~mm}, \mathrm{y}=11.89 \mathrm{~mm}, \mathrm{z}=11.71 \mathrm{~mm}$ ) was applied to 3125 "assigned images." Therefore, the SPECT-like spatial resolution images (we defined these images as "blurred images") generated the same number as the "assigned image."

\section{2-3-3. Process No. 3 of the proposed method: Generating the "difference image" (Fig. 4)} We defined the image, obtained by the actual SPECT imaging, and normalized it with the maximum value, as the "real image." The voxel-by-voxel differences between the "blurred image" generated in Process No. 2 and the "real image" were calculated. Thereby, the subtracted images between the "real image" and the "blurred image" (we defined these subtracted images as the "difference image") generated the same number as the "blurred image."

2-3-4. Process No. 4 of the proposed method: Extracting the "difference image" with the minimum summed value and determining the accumulation amounts in each region (Fig. 5) 
The "difference image" with the minimum summed value was extracted from 3125 "difference images" obtained by Process No. 3. In other words, the "difference image" was extracted when the "blurred image" was approximated most to the "real image." We defined the assumed accumulation amounts, assigned to the "blurred image" used when the extracted "difference image" was created, as the accumulation amounts in the left and right caudate/putamen and the BG regions.

\section{2-3-5. Process No. 5 of the proposed method: Updating the assumed accumulation} amounts (Fig. 6)

The accumulation amounts in the left and right caudate/putamen, and BG regions obtained by Process No. 4 were obtained from the approximately assumed accumulation amounts, 0 , $0.5,1.0,1.5$, and 2.0 (the range of the assumed accumulation amounts was $0-2.0$, and the interval of the assumed accumulation amounts was 0.5). Therefore, the assumed accumulation amounts were updated in detail to examine the "blurred image" that approximated more to the "real image." In this update, the range and interval of the assumed accumulation amounts assigned in the second step were updated to half of the range and interval of the assumed accumulation amounts assigned in the first step of Process No. 2. For example, it hypothesizes that the "blurred image" is approximated most to the "real image" when 1.0 as the assumed accumulation amount is assigned to a certain region. Among the assumed accumulation amounts $(0,0.5,1.0,1.5,2.0)$ assigned in the first step, the values before and after the 1.0, which is the value when the "blurred image" is approximated most to the "real image" are determined as the range of the assumed accumulation amounts assigned in the second step. In other words, the range of the assumed accumulation amounts assigned in the second step is 0.5 to 1.5 . Moreover, although the interval of the assumed accumulation amounts $0,0.5,1.0,1.5$, and 2.0 is 0.5 in the first step, the interval is 0.25 in the second step, which is half of the interval in the first step. Therefore, in the second step, the five values $0.50,0.75,1.00,1.25$, and 1.50 , as the assumed accumulation amounts are reassigned to the same region. We performed an exception process in case the "blurred image" was approximated most to the "real image," when the assumed accumulation amount was zero, since the process in the proposed method was performed so that the assumed accumulation amount was not negative. In case the "blurred image" is approximated most to 
the "real image" when the assumed accumulation amount is 0 , the range of the assumed

218 accumulation amount assigned in the second step is not the value before and after 0 , which 219 is assigned in the first step, and as an exception, only the value after the 0 is used. In other 220 words, the range of the assumed accumulation amounts assigned in the second step will be 221 from 0 to 0.50 in this update. However, the interval of the assumed accumulation amounts is 2220.25 , as with the usual process. Therefore, three values, $0,0.25,0.50$, as the assumed accumulation amounts are assigned again in the second step in case the "blurred image" is most approximated to the "real image" when the assumed accumulation amount is 0 . This update of the assumed accumulation amounts was performed in all the five regions (the left and right caudate/putamen and BG regions). Subsequently, the process from No. 2 to No. 5 was iterated. This series of Process No. 2-No. 5 was performed 10 times.

2-3-6. Process No. 6 of the proposed method: Determining the final accumulation amounts for each region and calculation of indices (Fig. 7) After Process No. 2-No. 5 was performed 10 times, the final "blurred image," which used for generating the "difference image" indicated the minimum summed value, was extracted. In other words, the "blurred image," which was approximated most to the "real image" in all generated "blurred images," was extracted. We subsequently defined this final extracted "blurred image" as the "generated image." This obtained "generated image" was undone before a 3D Gaussian filter was applied, i.e., the "assigned image" corresponded to the "generated image," was extracted. This "assigned image" obtained by this process was the ideal image with no blur reflecting the accumulation amounts in the left and right caudate/putamen and BG regions, and we defined this image as the "ideal assigned image." We finally determined the assumed accumulation amounts, assigned to the left and right caudate/putamen and the BG regions when the "ideal assigned image" was created, as the accumulation amounts for each region. The final accumulation amount obtained by the proposed method was determined as SPECT count density by the proposed method. We computed the SBR and caudate-putamen ratio (CPR) following the formula using SPECT count density by the 


$$
\mathrm{CPR}=\frac{\text { Caudate binding-BG binding }}{\text { Putamen binding-BG binding }}
$$

\section{2-4. Comparison method}

250 To represent the validity of the proposed method, as a comparison with the proposed method, we computed the SPECT count density using the comparison method. In the comparison method, the SPECT count density was calculated using the VOls for the left and right caudate/putamen and BG regions, which were applied from the CT images to the SPECT images in Process No. 1. Since Process No. 2 and the subsequent in proposed methods were not performed in the comparison method, PVE correction (PVC) was not performed in the SPECT count density calculated by the comparison method, which was different from the proposed method. Therefore, we defined the SPECT count density calculated by the comparison method as SPECT count density without PVC.

\section{2-5. Evaluation method}

We measured the actual count density (count/s $\cdot g$ ) of the ${ }^{123}$ solution filled into the striatal phantom by an auto-well counter. The correlation between the actual count density and the SPECT count density by the proposed method was evaluated using the Pearson's correlation coefficient. In addition, the correlation coefficient between the actual count density and the SPECT count density $_{\text {without Pvc }}$ was also calculated. The difference between these two correlation coefficients was tested using the Meng-Rosenthal-Rubin method [9].

In "Phantom2," we defined SBR and CPR, calculated by the actual count density of

${ }^{123}$ I solution filled into the striatal phantom, as each theoretical value. The absolute errors between the SBR calculated by the proposed method and the theoretical value were calculated using the following formula (the absolute errors for CPR were also calculated): The absolute error $=$ The value calculated by the proposed method - theoretical value(3)

\section{Results}

274 The comparison between the "real image" acquired by the actual SPECT imaging and the 275 "generated image" obtained by the proposed method is represented in Fig. 8. The images 276 shown in Fig. 8 were in the same display condition, and the counts and contrasts of the 
caudate, putamen and the BG in the "generated image" were visually similar to them in the "real image."

The correlation coefficient between the actual count density filled into the phantom

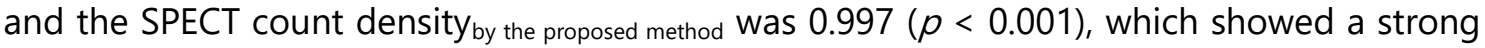
positive correlation (Fig. 9a). In addition, the correlation coefficient between the actual count density and the SPECT count density without PVC $_{\text {was }} 0.973(p<0.001)$, which also showed a strong correlation (Fig. 9b). Upon significant difference testing between the two correlation coefficients, the correlation of the proposed method was significantly higher than that of the comparison method $(p<0.001)$.

In "Phantom2," the errors between the SBR calculated by the proposed method and the theoretical SBR, calculated by the actual count density of ${ }^{123}$ | solution filled into the phantom are shown in Table 3 The SBR calculated by the proposed method was overestimated in the left and right caudate/putamen regions, that is, all the regions. In addition, the absolute errors between the CPR calculated by the proposed method and the theoretical CPR were approximately 0.1 , and the CPRs calculated by the proposed method approached the theoretical CPRs (Table 4).

\section{Discussion}

From a pathological viewpoint in PD, it is desirable to evaluate the accumulation amounts in the caudate and putamen through DaT SPECT. However, it is difficult to separate the caudate and putamen owing to the low spatial resolution of the SPECT device. It is also difficult to accurately evaluate the accumulation amounts due to PVE. Therefore, this study aimed to verify whether the newly proposed method could accurately evaluate the accumulation amounts in the caudate and putamen, with reduced PVE.

As shown in Fig. 8, the counts and contrasts of the caudate, the putamen, and the BG in the "generated image" were visually similar to those in the "real image" for the same display condition. In addition, the SPECT count density by the proposed method strongly correlated with the actual count density of the ${ }^{123}$ I solution filled into the phantom (Fig. 9a). This finding of the correlation between true values and measured values was comparable to other previously reported study [10]. Similarly, there was a strong correlation between the actual

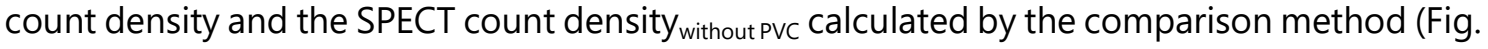


9b). However, the correlation coefficient of the proposed method was significantly higher than that of the comparison method $(p<0.001)$. The SPECT count density by the proposed method was an obtained value that considered the influence of PVE. As a result, it approached an accurate accumulation amount, and thus, a stronger correlation in the proposed method was considered. However, the SBRs calculated by the proposed method were overestimated in all the regions (Table 3). This overestimation is considered since the contrast between the striatum and the BG was overemphasized due to over-correction produced by $\mathrm{SC}, \mathrm{AC}$, and PVC. It has been reported that the SC and the AC improve the quantitative evaluation compared to no corrections [11-14]. In addition to the SC and AC, PVC also has been reported to be valuable in quantitative evaluation $[13,14]$. Although the SBR calculated by the proposed method is overestimated by the over-correction, it is considered since the SBRs calculated by the proposed method did not indicate the absolute theoretical SBRs calculated by the actual count density of ${ }^{123}$ I solution filled into the phantom. However, since the SPECT count density $y_{\text {by the proposed method }}$ strongly correlated with the actual count density of ${ }^{123}$ | solution filled into the phantom, it seems that the SBRs calculated by the proposed method represent not the absolute theoretical SBR, but the relative theoretical SBR on SPECT image. In support of this, the CPRs obtained by the proposed method were extremely close to the absolute theoretical CPR calculated by the actual count density of the ${ }^{123}$ solution filled in the phantom (Table 4). In the proposed method, the SPECT count densities of the caudate and the putamen were increased by the corrections; however, the caudate was divided by the putamen in the $\mathrm{CPR}$, which indicates that the influence of the corrections was canceled. Therefore, the CPRs calculated by the proposed method were nearing the absolute CPRs calculated by the actual count density of the ${ }^{123}$ I solution filled into the phantom. In PD, the accumulation begins to decrease from the putamen [6]. Hence, for evaluating the accurate CPR, the proposed method can detect the accumulation reduction in the putamen with high sensitivity, which is expected to be beneficial in clinical practice.

In the present study, when examining the "blurred image" approximated to the "real image," the process where the assumed accumulation amounts were updated in detail to examine the "blurred image" approximated more to the "real image," was performed. This update process was performed 10 times. The variability rate of SBR with the number of processes was calculated using the following formula: 
$340 \mathrm{SBR}_{\mathrm{n}}$ represents the SBR in the nth process. The variability rate of CPR was also calculated using Eq. (4). The variability rate of SBR and CPR with the number of processes is shown in

342 Fig.10. We considered SBR and CPR to converge when the number of processes was over seven since the variability rates of both SBR and CPR were less than $5 \%$. Therefore, 10 times, which was the number of processes used in the present study, was sufficient to converge.

In the present study, we only examined the use of a phantom. Therefore, it is necessary to apply the proposed method in clinical practice and examine the utility of this method in further research. By using the anatomical position information obtained from the morphological image, such as the MR image and applying the proposed method, it is possible to calculate the accumulation amounts in each region of the brain considering the PVE. It has been reported that the regions of ${ }^{123}$ I-FP-CIT low accumulation, such as the cerebral ventricle, have an effect on the SBR calculated by the method using the large VOI, as in the Southampton method $[15,16]$. In the proposed method, it is possible to exclude the influence on the SBR of the cerebral ventricle by performing a calculation process that uses multiple regions such as specific regions (the caudate and putamen), non-specific regions (the occipital lobe, cerebellum, and whole brain), and the region of ${ }^{123}$ I-FP-CIT low accumulation (the cerebral ventricle). In addition, the influences of the mutual counts between the regions i.e. spill-in and spill-out was considered in the proposed method. To date, various methods have been reported to calculate the semi-quantification index by processing images obtained by the SPECT imaging or devising analysis method. Templatebase method [17,18] or CT/MR-guide method [19] can compute semi-quantification index considering with the morphology of the caudate and putamen. However, these methods cannot eliminate the PVE. In addition, it is difficult to grasp the individual patient morphology in the method using only SPECT images such as template-base method. It is desirable to use an anatomical scan (CT or MR) especially in the case of severe loss of accumulation [20]. A previous study has reported that striatal volume reduces with ageing [21]. Fixed-VOI method (the methods calculate semi-quantification index using fixed striatal volume) such as 367 Southampton method tend to be overestimated especially in older age [22,23]. Therefore, it 368 is important to include the individual patient morphology. The difficulty in evaluating the 
caudate and putamen, separately owing to the low spatial resolution of the SPECT images makes the border between the caudate and putamen vague. Even when the caudate and putamen can be separated by the combined use of the morphological images, the counts leaking from the caudate and putamen (spill-out) enter each other's region (spill-in), making the accumulation amounts in each region ambiguous. Moreover, the counts leaking from the regions other than the caudate and putamen also enter the caudate and putamen regions (spill-in). Therefore, it is extremely difficult to calculate the accurate accumulation amounts in the caudate and putamen using SPECT images obtained by imaging. The BasGan method [10] can compute the accumulation of the caudate and putamen separately with consideration of PVE. The PVC method used in this method has been previously reported [24]. This PVC method only uses the information included in the VOls. The calculation process of the proposed method uses reverse direction approach, which is different from the calculation process that is normally used. In the proposed method, based on the anatomical position information by a morphological image and the spatial resolution information of the SPECT device, the calculation process involves examining the assumed accumulation amounts in each region of the brain to approach the SPECT image obtained by actual imaging, and uses this assumed accumulation amount to calculate the SBR. Therefore, different from the PVC method using in BasGan method, the proposed method uses the all information of SPECT images obtained by actual imaging for performing PVC. Therefore, since the proposed method examines the realistic accumulation using the all information of SPECT images obtained by actual imaging, leading to consideration of the counts leaked from the caudate and putamen (spill-out), and the counts entered into the caudate and putamen from regions other than the caudate and putamen (spill-in). Thus, it is expected that the proposed method can evaluate the accumulation amounts in the caudate and putamen more accurately.

This study has some limitations. The primary limitation of this study is that it examined only the phantom data in this study. In the present study, the image, which was approximated most to the SPECT image obtained by imaging, was examined by generating tens of thousands of SPECT-like images. As a result, the process took a few hours, which is not suitable for clinical practice. Moreover, the accumulation amounts in the VOI were assumed to be uniform in the proposed method. The voxel-by-voxel process allows the consideration of the non-uniformity of the accumulation amounts. However, since an 
400

401

402

403

404

405

406

407

408

409

410

411

412

413

414

415

416

417

418

419

420

421

422

423

424

425

426

427

428

429

enormous amount of processing is required, it was not performed in the present study. By using an optimization method that minimizes the difference between the SPECT image obtained by imaging and the generated SPECT-like image, the process takes lesser time, and it is possible to apply the proposed method in clinical practice. In the present study, the reason for not using the optimization method was that it was expected that the differences in the optimization method or the parameter would lead to different results. Hence, an exhaustive method was implemented to examine the usefulness of the proposed method in a pure state without any influence other than the calculation process. If the process takes lesser time by using the optimization method in the future, it is expected possible to perform the voxel-by-voxel process and calculate the accumulation amounts in the detailed regions of the brain. We consider that in the future this leads in the computation of more accurate accumulation in detailed regions of the brain, considering the non-uniformity.

\section{Conclusion}

Using the newly proposed method, the accumulation amounts in the caudate and the putamen separately with the consideration of PVE could be calculated. We were able to prove the validity of the proposed method. However, in this study, the validity of the proposed method only examined on the phantom data. Therefore, further research involving examination in clinical practice is warranted.

(1)

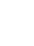

.

(1)


430 DaT: Dopamine transporter; ${ }^{123}\left|-F P-C I T:{ }^{123}\right|-N-\omega$-fluoropropyl-2 $\beta$-carboxymethoxy-3 $\beta$-(4431 iodophenyl)nortropane; PD: Parkinson disease; DLB: Dementia with Lewy bodies; SBR: Specific 432 binding ratio; SPECT: Single photon emission computed tomography; PVE: Partial volume 433 effect; VOI: Volume of interest; LMEGP: Low medium energy general purpose; 3D-OSEM: 3434 dimensional ordered subset expectation maximization method; FWHM: Full width at half 435 maximum; AC: attenuation correction; CT: Computed tomography; SC: scatter correction; 436 TEW: triple energy window; BG: Background; CPR: Caudate-putamen ratio; PVC: PVE 437 correction

438

439 Ethics approval and consent to participate

$440 \quad$ Not applicable

441

442 Consent for publication

443 Not applicable

444

445 Availability of data and material

446 The datasets used and/or analysed during the current study are available from the 447 corresponding author on reasonable request.

448

449 Competing interests

450 The authors declare that they have no competing interests.

451

452 Funding

453 This study was not funded by any funding bodies.

454

455 Authors' contributions

$456 \mathrm{KK}$ is the guarantor of integrity of the entire study. $\mathrm{YI}, \mathrm{NF}$, and $\mathrm{KK}$ contributed to the 457 conception and design of the study. YI, NF, TT, SA and KK contributed to the acquisition of 458 data. YI contributed to the analysis of data. YI, NF and KK contributed to the manuscription 459 preparation. NF, KH, SA, MK, SNand KK contributed to the manuscript editing. All authors 460 read and approved the final manuscript. 


\section{Acknowledgements}

463 The authors would like to thank the staff of the Department of Nuclear Medicine, Nagoya 464 University Hospital for their valuable support. The authors thank Yoshiyuki Hirano, 465 Department of Integrated Health Sciences, Nagoya University Graduate School of Medicine, 466 for his valuable support.

467

468

\section{References}

469 [1] Booij J, Tissingh G, Boer GJ, Speelman JD, Stoof JC, Janssen AGM, et al. I-123 FP-CIT SPECT 470 shows a pronounced decline of striatal dopamine transporter labelling in early and advanced 471 Parkinson's disease. Journal of Neurology Neurosurgery and Psychiatry. 1997;62:133-40.

472 [2] Tatsch K, Poepperl G. Nigrostriatal Dopamine Terminal Imaging with Dopamine 473 Transporter SPECT: An Update. Journal of Nuclear Medicine. 2013;54:1331-8.

474 [3] Walker Z, Costa DC, Walker RWH, Shaw K, Gacinovic S, Stevens T, et al. Differentiation of 475 dementia with Lewy bodies from Alzheimer's disease using a dopaminergic presynaptic 476 ligand. Journal of Neurology Neurosurgery and Psychiatry. 2002;73:134-40.

477 [4] Soret M, Koulibaly PM, Darcourt J, Hapdey S, Buvat I. Quantitative accuracy of 478 dopaminergic neurotransmission imaging with (123)I SPECT. Journal of Nuclear Medicine. 479 2003;44:1184-93.

480 [5] Tossici-Bolt L, Hoffmann SMA, Kemp PM, Mehta RL, Fleming JS. Quantification of I-123 481 FP-CIT SPECT brain images: an accurate technique for measurement of the specific binding 482 ratio. European Journal of Nuclear Medicine and Molecular Imaging. 2006;33:1491-9.

483 [6] Fearnley JM, Lees AJ. AGING AND PARKINSONS-DISEASE - SUBSTANTIA-NIGRA 484 REGIONAL SELECTIVITY. Brain. 1991;114:2283-301.

485 [7] Walker Z, Costa DC, Walker RWH, Lee L, Livingston G, Jaros E, et al. Striatal dopamine 486 transporter in dementia with Lewy bodies and Parkinson disease - A comparison. Neurology. $487 \quad 2004 ; 62: 1568-72$.

488 [8] O'Brien JT, Colloby S, Fenwick J, Williams ED, Firbank M, Burn D, et al. Dopamine 489 transporter loss visualized with FP-CIT SPECT in the differential diagnosis of dementia with 490 Lewy bodies. Archives of Neurology. 2004;61:919-25. 

COEFFICIENTS. Psychological Bulletin. 1992;111:172-5.

493 [10] Calvini P, Rodriguez G, Inguglia F, Mignone A, Guerra UP, Nobili F. The basal ganglia matching tools package for striatal uptake semi-quantification: description and validation. European Journal of Nuclear Medicine and Molecular Imaging. 2007;34:1240-53. [11] Furuta A, Onishi H, Yamaki N, Yada N, Amijima H. Impact of quantitative index derived from 123I-FP-CIT-SPECT on reconstruction with correction methods evaluated using a 3Dstriatum digital brain phantom. Radiological Physics and Technology. 2018;11:294-302. [12] Tossici-Bolt L, Dickson JC, Sera T, de Nijs R, Bagnara MC, Jonsson C, et al. Calibration of gamma camera systems for a multicentre European I-123-FP-CIT SPECT normal database. European Journal of Nuclear Medicine and Molecular Imaging. 2011;38:1529-40.

502 [13] Soret M, Koulibaly PM, Darcourt J, Hapdey S, Buvat I. Quantitative accuracy of dopaminergic neurotransmission imaging with (123)I SPECT. Journal of Nuclear Medicine. 2003;44:1184-93.

[14] Soret M, Koulibaly PM, Darcourt J, Buvat I. Partial volume effect correction in SPECT for striatal uptake measurements in patients with neurodegenerative diseases: impact upon patient classification. European Journal of Nuclear Medicine and Molecular Imaging. 2006;33:1062-72.

[15] Nonokuma M, Kuwabara Y, Hida K, Tani T, Takano K, Yoshimitsu K. Optimal ROI setting on the anatomically normalized I-123 FP-CIT images using high-resolution SPECT. Annals of

511 Nuclear Medicine. 2016;30:637-44.

512 [16] Mizumura S, Nishikawa K, Murata A, Yoshimura K, Ishii N, Kokubo T, et al. Improvement 513 in the measurement error of the specific binding ratio in dopamine transporter SPECT 514 imaging due to exclusion of the cerebrospinal fluid fraction using the threshold of voxel RI 515 count. Annals of Nuclear Medicine. 2018;32:288-96.

516 [17] Matesan M, Gaddikeri S, Longfellow K, Miyaoka R, Elojeimy S, Elman S, et al. I-123

517 DaTscan SPECT Brain Imaging in Parkinsonian Syndromes: Utility of the Putamen-to-

518 Caudate Ratio. Journal of Neuroimaging. 2018;28:629-34.

519 [18] Koch W, Radau PE, Hamann C, Tatsch K. Clinical testing of an optimized software

520 solution for an automated, observer-independent evaluation of dopamine transporter 521 SPECT studies. Journal of Nuclear Medicine. 2005;46:1109-18. 
522 [19] Hsu CC, Chang YH, Lin WC, Tang SW, Wang PW, Huang YC, et al. The feasibility of using

523 CT-guided ROI for semiquantifying striatal dopamine transporter availability in a hybrid

524 SPECT/CT system. ScientificWorldJournal. 2014;2014:879497.

525 [20] Morbelli S, Esposito G, Arbizu J, Barthel H, Boellaard R, Bohnen NI, et al. EANM practice

526 guideline/SNMMI procedure standard for dopaminergic imaging in Parkinsonian

527 syndromes 1.0. European Journal of Nuclear Medicine and Molecular Imaging.

$528 \quad 2020 ; 47: 1885-912$.

529 [21] Raz N, Rodrigue KM, Kennedy KM, Head D, Gunning-Dixon F, Acker JD. Differential

530 aging of the human striatum: Longitudinal evidence. American Journal of Neuroradiology.

$531 \quad 2003 ; 24: 1849-56$.

532 [22] Roberts G, Lloyd JJ, Petrides GS, Donaghy PC, Kane JP, Durcan R, et al. 123 I-FP-CIT

533 striatal binding ratios do not decrease signifcantly with age in older adults. Annals of

534 Nuclear Medicine. 2019;33:434-43.

535 [23] Matsuda H, Murata M, Mukai Y, Sako K, Ono H, Toyama H, et al. Japanese multicenter

536 database of healthy controls for I-123 FP-CIT SPECT. European Journal of Nuclear Medicine

537 and Molecular Imaging. 2018;45:1405-16.

538 [24] Koole M, Laere KV, Van de Walle R, Vandenberghe S, Bouwens L, Lemahieu I, et al. MRI

539 guided segmentation and quantification of SPECT images of the basal ganglia: a phantom

540 study. Computerized Medical Imaging and Graphics. 2001;25:165-72.

541

542

543

544

545

546

547

548

549

550 Fig. 1 Flow of the calculation process by the proposed method

551 The positional information of the left and right caudate/putamen, and the background (BG)

552 regions is obtained on computed tomography images. Based on this positional information, 
the assumed accumulation amounts are assigned into each region, and the images with no

554 blur, called the "assigned image," were generated. A three-dimensional Gaussian filter 555 equivalent to SPECT spatial resolution in clinical condition was applied to the "assigned 556 image," and the single-photon emission computed tomography (SPECT)-like images, called

557 the "blurred image," was generated. The voxel-by-voxel differences between the actual image 558 obtained by imaging ("real image") and "blurred image" were calculated, and the "difference 559 image" was generated. The "difference image" with the minimum summed values was 560 extracted, and the assumed accumulation amounts, assigned into the "blurred image" used 561 when generating the extracted "difference image," were determined as the accumulation 562 amounts in the left and right caudate/putamen and BG regions. The assumed accumulation 563 amounts assigned to each region were updated in detail, and Process No. 2 was performed again. After all the 10 times of a series of Process No. 2-No. 5 were performed, the final accumulation amounts in the left and right caudate/putamen and BG regions were determined, and specific binding ratio (SBR) and the caudate-putamen ratio (CPR) were 567 calculated.

568

Fig. 2 Process No. 1 of the proposed method: Extracting the positional information of each region

571 Using the PMOD software, the volume of interests (VOIs) were manually established along

572 the morphology of the left and right caudate/putamen and background (BG) regions on the 573 computed tomography (CT) images. The VOls established on the CT images were applied to 574 the single-photon emission computed tomography (SPECT) images. Using the VOIs on SPECT 575 images, the mask images extracted the positional information of the left and right 576 caudate/putamen and BG regions.

577

578 Fig. 3 Process No. 2 of the proposed method: Generating the "assigned image" and "blurred 579 image"

580 Based on the mask images obtained from Process No. 1, the assumed accumulation amounts $581(0,0.5,1.0,1.5$, and 2.0) were assigned to the five regions (i.e., the left and right 582 caudate/putamen and background $[B G]$ regions). Since the five values $(0,0.5,1.0,1.5,2.0)$ 583 were assigned to the five regions (the left and right caudate/putamen and BG regions), $5^{5}$ 
584 (=3125) images with no blur (these images are called the "assigned image") were generated.

585 A three-dimensional Gaussian filter equivalent to single-photon emission computed 586 tomography (SPECT) spatial resolution in clinical condition was applied to the "assigned 587 image." Thus, 3125 SPECT-like spatial resolution images (these images were called the 588 "blurred image") were generated.

589

590 Fig. 4 Process No. 3 of the proposed method: Generating the "difference image"

591 The image obtained by actual single-photon emission computed tomography (SPECT)

592 imaging and normalized with the maximum value was defined as the "real image." The voxel-

593 by-voxel differences between the "real image" obtained by actual imaging and the "blurred

594 image" obtained by Process No. 2 were calculated, and 3125 "difference images" were 595 generated.

596

Fig. 5 Process No. 4 of the proposed method: Determining the accumulation amounts in each 598 region

599 The "difference image" with the minimum summed values was extracted among 3125 600 "difference images" obtained by Process No. 3. The "difference image" with the minimum 601 summed values means that "blurred image" is most approximated to the "real image." The 602 assumed accumulation amounts in the left and right caudate/putamen and background (BG) 603 regions, assigned to the "blurred image" used when generating the extracted "difference 604 image," were determined as the accumulation amounts in each region.

605

606 Fig. 6 Process No. 5 of the proposed method: Updating the assumed accumulation amounts 607 The accumulation amounts obtained from Process No. 4 were calculated from the roughly 608 assumed accumulation amounts. Therefore, the assumed accumulation amounts were 609 updated in more detail to examine the image that was more approximated to the "real image." 610 For example, it hypothesizes that the "blurred image" was most approximated to the "real 611 image" when the assumed accumulation amount in a certain region was 1.0. Then, among 612 the assumed accumulation amounts $(0,0.5,1.0,1.5$, and 2.0) assigned in the first step, the 613 values before and after the 1.0, which is the value when the "blurred image" was most 614 approximated to the "real image," are determined as the range of the assumed accumulation 
amounts assigned in the second step. In other words, the range of the assumed accumulation amounts assigned in the second step is 0.5 to 1.5 . Moreover, although the interval of the

617 assumed accumulation amounts $0,0.5,1.0,1.5$, and 2.0 is 0.5 in the first step, the interval is 6180.25 in the second step, which is half of the interval in the first step. Therefore, in the second 619 step, the five values $0.50,0.75,1.00,1.25$, and 1.50, as the assumed accumulation amounts 620 are assigned to the same region again. This update of the assumed accumulation amount was performed in all the five regions (the left and right caudate/putamen and background [BG] regions). Subsequently, the process from No. 2 to No. 5 was iterated. This series of Process No. 2-No. 5 was performed 10 times.

Fig. 7 Process No. 6 of the proposed method: Determining the final accumulation amounts and calculating indices After performing the series in Process No. 2-No. 510 times, the "blurred image" finally most approximated to the "real image" was defined as the "generated image." The "generated image" was returned to the image which was before the three-dimensional Gaussian filter was applied in Process No. 2. This ideal image with no blurs was defined as the "ideal assigned image." The assumed accumulation amounts in the left and right caudate/putamen and background (BG) regions assigned into the "ideal assigned image" were determined as the accumulation amounts in each region. Using these values, the specific binding ratio (SBR) and the caudate-putamen ratio (CPR) were calculated.

Fig. 8 Comparison between the "real image" and "generated image"

637 The "Real image" was the image obtained by the actual imaging and the "generated image" 638 was the final image obtained by the proposed method. (a) shows the "real image" and 639 "generated image" obtained from "Phantom1" and (b) shows the image obtained by "Phantom2." These images were in the same display condition for comparison between the "real image" and the "generated image." The counts and contrasts of the caudate, putamen, and background (BG) in the "generated image" were visually similar to those in the "real image." 
645 Fig. 9 Correlation between the actual count density and the single-photon emission 646 computed tomography (SPECT) count density

647 This figure shows the correlation between the actual count density of the ${ }^{123}$ / solution filled 648 in the phantom and the SPECT count density. (a) shows the correlation between the actual 649 count density and SPECT count density by the proposed method. The SPECT count density by the proposed 650 method significantly correlated with the actual count density $(r=0.997, p<0.001)$. (b) shows the

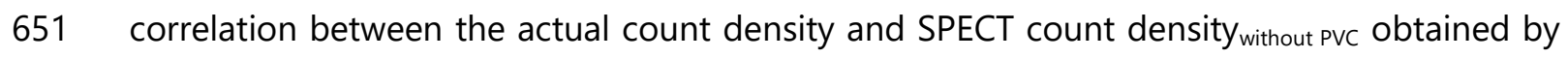
652 the comparison method. The SPECT count density without PVc significantly correlated with the 653 actual count density $(r=0.973, p<0.001)$.

654

655 Fig. 10 The relationship between the number of processing times and the variability rate

656 (a) shows the relation between the number of processing times and the variability of specific 657 binding ratio (SBR). When the number of processing times was low, SBRs were greatly variable. 658 However, SBRs were converged as the processing was repeated. When the number of 659 processing times was more than seven, the variability rates of the SBR were less than 5\%. (b) 660 shows the relation between the number of processing times and the variability of 661 caudate/putamen ratio (CPR). This relation showed a similar trend to the SBR.

662

663

664

665

666

667

668

669

Table 1 Phantom data

674 The scheduled ratios show the radioactivity ratios of the ${ }^{123}$ solution scheduled before 675 creating the phantom. The actual count densities show that the ${ }^{123}$ count density actually 
676 filled in the phantom was measured by an auto-well counter. The actual ratios show the ratio 677 calculated using the actual count density. In "Phantom1", ${ }^{123}$ | solution was only filled in the 678 left and right caudate, and the other regions (the left and right putamen, and background $679[B G]$ regions) were filled with water. In "Phantom2", the ${ }^{123}$ s solution was filled in all regions.

680

\begin{tabular}{crrrrr}
\hline & & & Phantom1 & & \\
& Right caudate & Right putamen & Left caudate & Left putamen & BG \\
\hline Scheduled ratio & 1.80 & 0.00 & 1.00 & 0.00 & 0.00 \\
Actual count density (count/s·g) & 25123.21 & 0.00 & 14156.71 & 0.00 & 0.00 \\
Actual ratio & 1.77 & 0.00 & 1.00 & 0.00 & 0.00 \\
\hline & & & Phantom2 & & BG \\
\hline Scheduled ratio & Right caudate & Right putamen & Left caudate & Left putamen & 1.00 \\
Actual count density (count/s·g) & 9.00 & 9.00 & 5.00 & 4.00 & 2337.02 \\
Actual ratio & 23777.24 & 23777.24 & 13398.26 & 11187.14 & 1.00 \\
\hline
\end{tabular}

681

682

683

684

685

686

687

688

689

690

691

692

693

694

695 Table 2 Terms used in the proposed method 
696 The "assigned image," "blurred image," "real image," "difference image," "generated image," 697 and "ideal assigned image" were coined words that were created to describe the proposed 698 method. The descriptions of these terms used in the proposed method are presented. 699

\begin{tabular}{ll}
\hline Term & Definition \\
\hline Assigned image & "Assigned image" is the ideal image with no blur that assumed accumulation amounts is \\
& assigned to each region (the left and right caudate/putamen, and BG regions). \\
"Blurred image" is the SPECT-like image that 3D Gaussian filter equivalent to SPECT spatial & \\
Blurred image & "Resolution under clinical condition is applied to "assigned image". \\
Real image & maximum value. \\
"Difference image" is the image that the voxel-by-voxel difference between "blurred image" & and "real image" is calculated. \\
Difference image & "Generated image" is the "blurred image" that is approximated most to the "real image." \\
Generated image & "Ideal assigned image" is the "assigned image" which is before 3D Gaussian filter applied to \\
& "generated image".
\end{tabular}

700 
713 Table 3 Comparison between the theoretical specific binding ratio (SBR) and SBR obtained by

714 the proposed method

715 Theoretical SBRs are calculated by the actual count density of the ${ }^{123}$ solution filled in the

716 phantom. Calculated SBRs were calculated using the proposed method. Absolute errors

717 between the theoretical SBR and calculated SBR were positive in all regions, and the 718 calculated SBRs were overestimated in all regions.

719

\begin{tabular}{lrrrr}
\hline & Right caudate & Right putamen & Left caudate & Left putamen \\
\hline Theoretical SBR & 9.17 & 9.17 & 4.73 & 3.79 \\
Calculated SBR & 11.83 & 11.07 & 5.92 & 4.36 \\
Absolute error & 2.65 & 1.89 & 1.19 & 0.57 \\
\hline
\end{tabular}

720

721

722 Table 4 Comparison between the theoretical caudate-putamen ratio (CPR) and CPR obtained 723 by the proposed method

724 Theoretical CPRs were calculated by the actual count density of the ${ }^{123}$ I solution filled in the 725 phantom. Calculated CPRs were calculated using the proposed method. Absolute errors 726 between the theoretical CPR and calculated CPR were almost 0 , and the calculated CPRs were 727 close to the theoretical CPR.

728

\begin{tabular}{ccc}
\hline & Right striatum & Left striatum \\
\hline Theoretical CPR & 1.00 & 1.25 \\
Calculated CPR & 1.07 & 1.36 \\
Absolute error & 0.07 & 0.11 \\
\hline
\end{tabular}




\section{Figures}

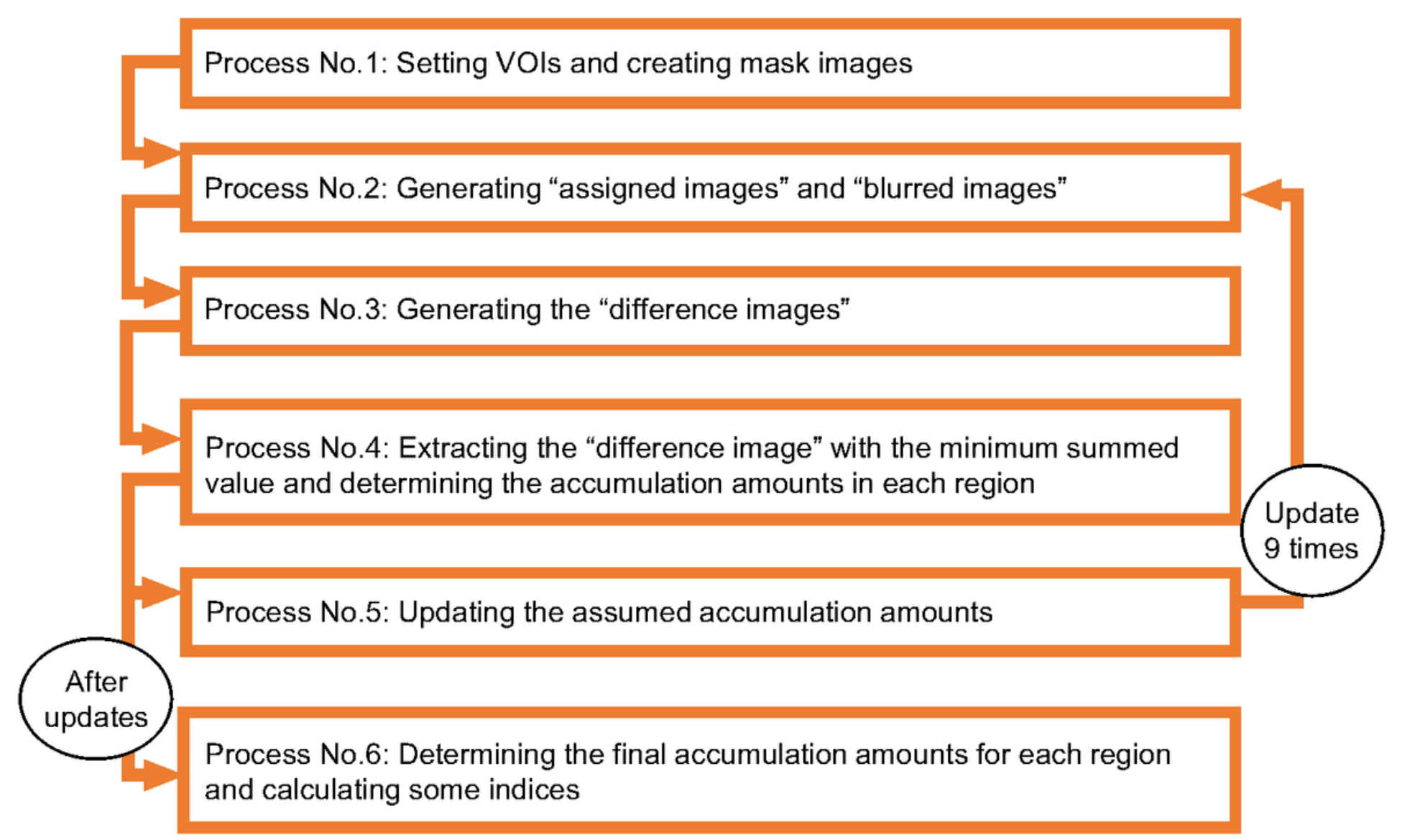

Figure 1

Flow of the calculation process by the proposed method The positional information of the left and right caudate/putamen, and the background (BG) regions is obtained on computed tomography images.

Based on this positional information, the assumed accumulation amounts are assigned into each region, and the images with no blur, called the "assigned image," were generated. A three-dimensional Gaussian filter equivalent to SPECT spatial resolution in clinical condition was applied to the "assigned image," and the single-photon emission computed tomography (SPECT)-like images, called the "blurred image," was generated. The voxel-by-voxel differences between the actual image obtained by imaging ("real image") and "blurred image" were calculated, and the "difference image" was generated. The "difference image" with the minimum summed values was extracted, and the assumed accumulation amounts, assigned into the "blurred image" used when generating the extracted "difference image," were determined as the accumulation amounts in the left and right caudate/putamen and BG regions. The assumed accumulation amounts assigned to each region were updated in detail, and Process No. 2 was performed again. After all the 10 times of a series of Process No. 2-No. 5 were performed, the final accumulation amounts in the left and right caudate/putamen and BG regions were determined, and specific binding ratio (SBR) and the caudate-putamen ratio (CPR) were calculated. 


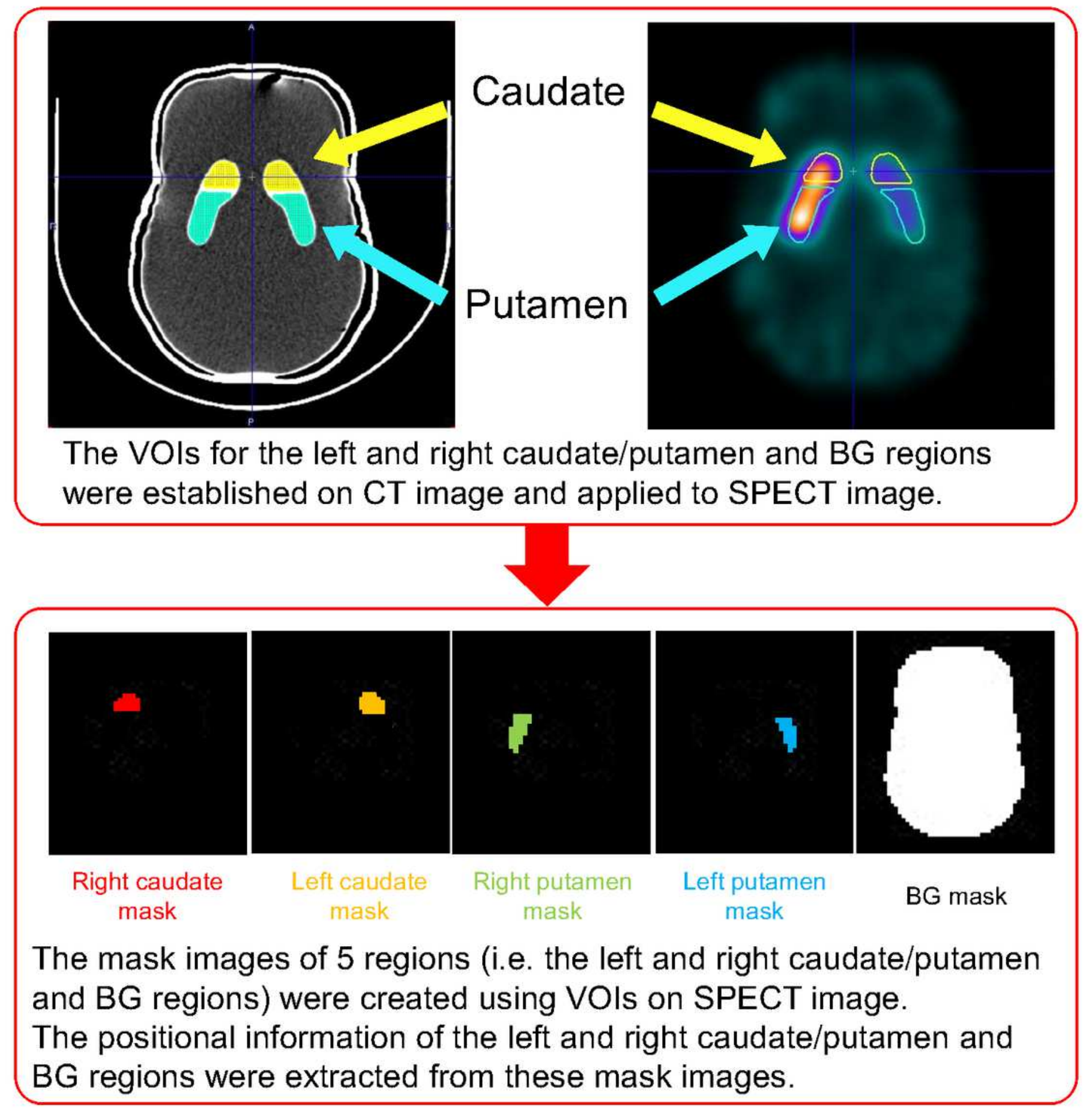

Figure 2

Process No. 1 of the proposed method: Extracting the positional information of each region Using the PMOD software, the volume of interests (VOIs) were manually established along the morphology of the left and right caudate/putamen and background (BG) regions on the computed tomography (CT) images. The VOls established on the CT images were applied to the single-photon emission computed tomography (SPECT) images. Using the VOIs on SPECT images, the mask images extracted the positional information of the left and right caudate/putamen and BG regions. 


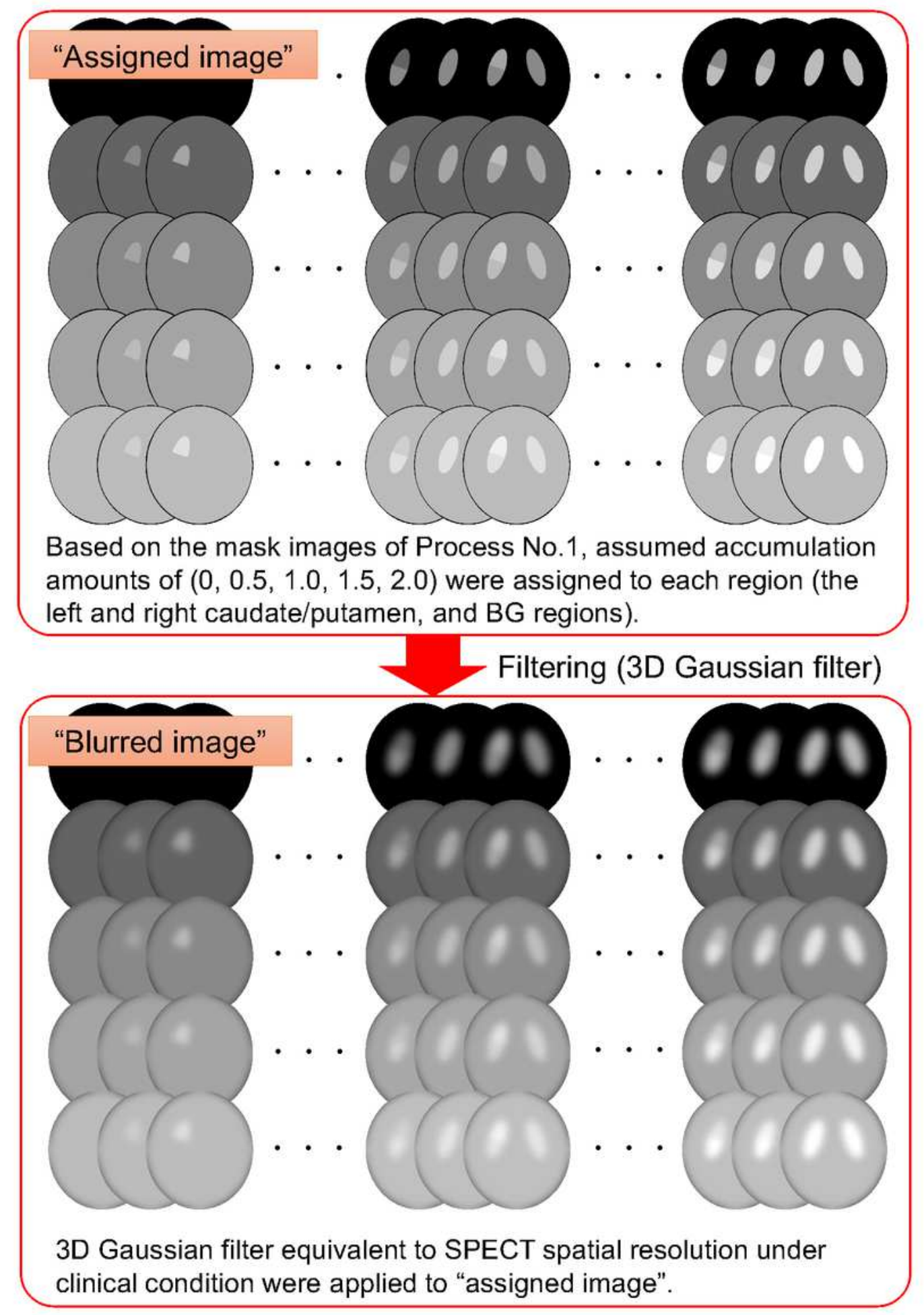

\section{Figure 3}

Process No. 2 of the proposed method: Generating the "assigned image" and "blurred image" Based on the mask images obtained from Process No. 1 , the assumed accumulation amounts $(0,0.5,1.0,1.5$, and 2.0) were assigned to the five regions (i.e., the left and right caudate/putamen and background [BG] regions). Since the five values $(0,0.5,1.0,1.5,2.0)$ were assigned to the five regions (the left and right caudate/putamen and BG regions), 55 (=3125) images with no blur (these images are called the 
"assigned image") were generated. A three-dimensional Gaussian filter equivalent to single-photon emission computed tomography (SPECT) spatial resolution in clinical condition was applied to the "assigned image." Thus, 3125 SPECT-like spatial resolution images (these images were called the "blurred image") were generated.

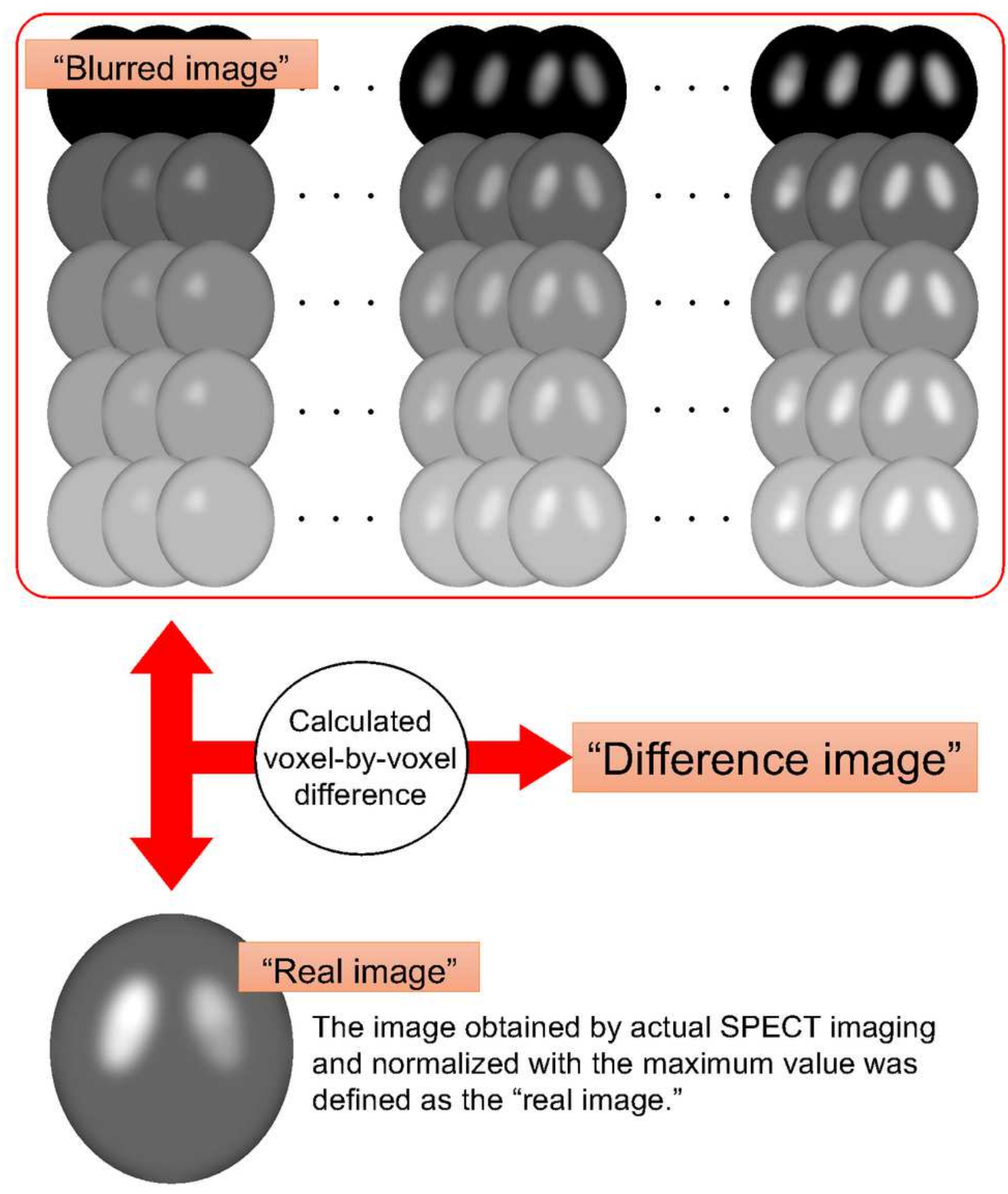

Figure 4 
Process No. 3 of the proposed method: Generating the "difference image" The image obtained by actual single-photon emission computed tomography (SPECT) imaging and normalized with the maximum value was defined as the "real image." The voxel-by-voxel differences between the "real image" obtained by actual imaging and the "blurred image" obtained by Process No. 2 were calculated, and 3125 "difference images" were generated.

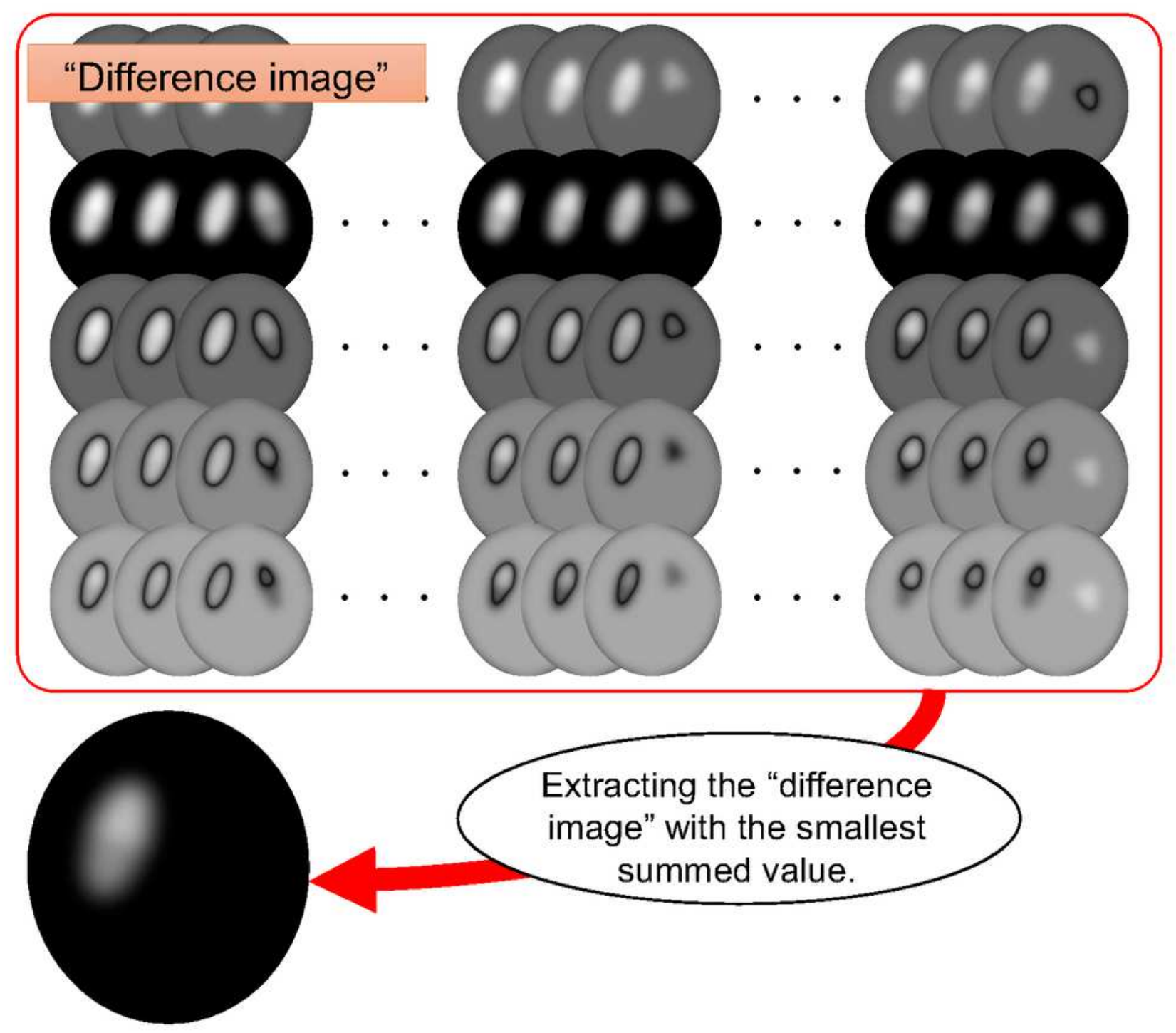

The assumed accumulation amounts, assigned to the "blurred image" used when generating the extracted "difference image," were determined as the accumulation amounts in the each region (the left and right caudate/putamen, and BG regions).

These accumulation amounts obtained in this process were updated in detail in Process No. 5. 
Process No. 4 of the proposed method: Determining the accumulation amounts in each region The "difference image" with the minimum summed values was extracted among 3125 "difference images" obtained by Process No. 3. The "difference image" with the minimum summed values means that "blurred image" is most approximated to the "real image." The assumed accumulation amounts in the left and right caudate/putamen and background (BG) regions, assigned to the "blurred image" used when generating the extracted "difference image," were determined as the accumulation amounts in each region.

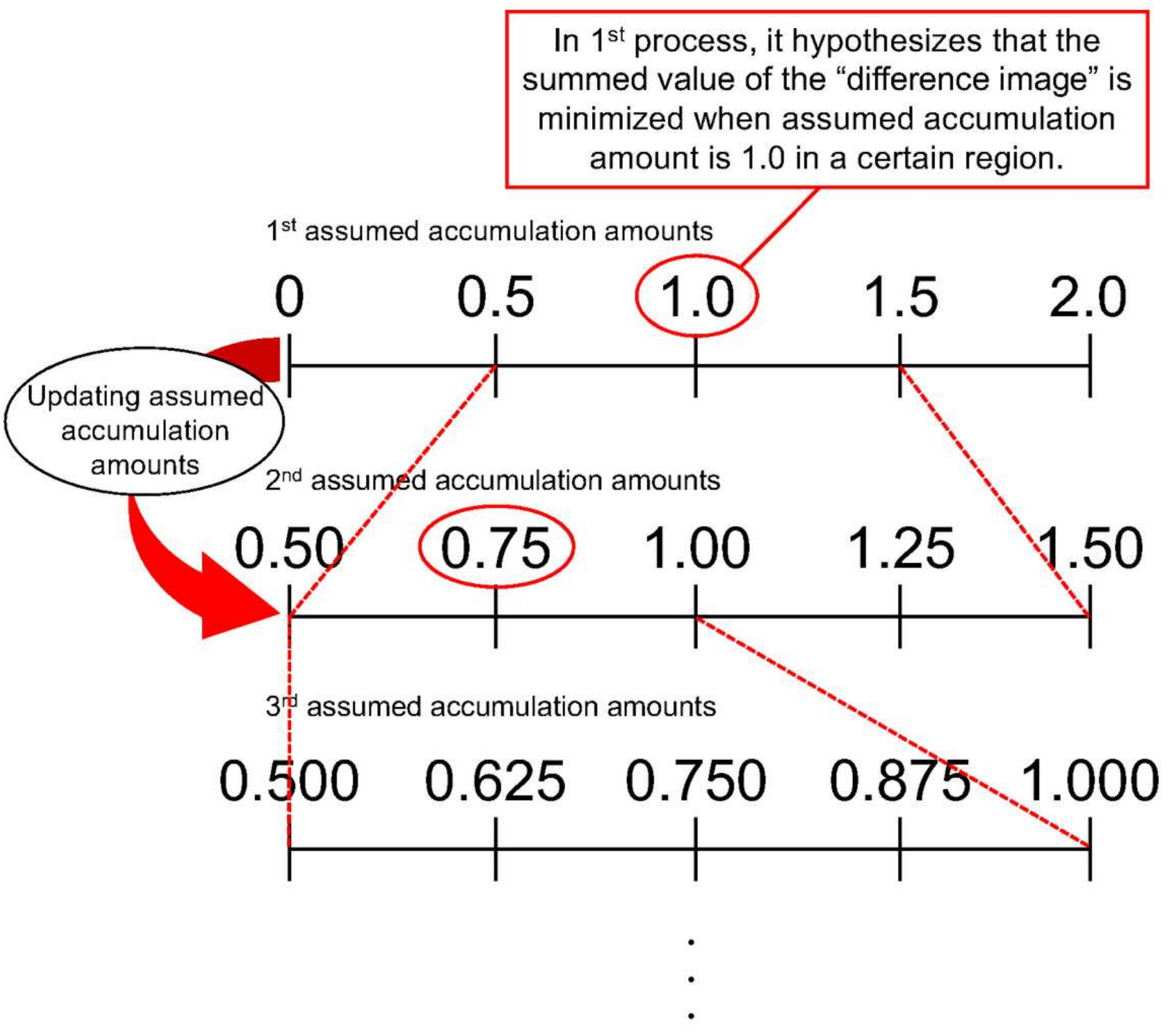

Obtaining until $10^{\text {th }}$ assumed accumulation amounts.

Figure 6

Process No. 5 of the proposed method: Updating the assumed accumulation amounts The accumulation amounts obtained from Process No. 4 were calculated from the roughly assumed accumulation 
amounts. Therefore, the assumed accumulation amounts were updated in more detail to examine the image that was more approximated to the "real image." For example, it hypothesizes that the "blurred image" was most approximated to the "real image" when the assumed accumulation amount in a certain region was 1.0. Then, among the assumed accumulation amounts $(0,0.5,1.0,1.5$, and 2.0$)$ assigned in the first step, the values before and after the 1.0, which is the value when the "blurred image" was most approximated to the "real image," are determined as the range of the assumed accumulation amounts assigned in the second step. In other words, the range of the assumed accumulation amounts assigned in the second step is 0.5 to 1.5. Moreover, although the interval of the assumed accumulation amounts 0 , $0.5,1.0,1.5$, and 2.0 is 0.5 in the first step, the interval is 0.25 in the second step, which is half of the interval in the first step. Therefore, in the second step, the five values $0.50,0.75,1.00,1.25$, and 1.50, as the assumed accumulation amounts are assigned to the same region again. This update of the assumed accumulation amount was performed in all the five regions (the left and right caudate/putamen and background [BG] regions). Subsequently, the process from No. 2 to No. 5 was iterated. This series of Process No. 2-No. 5 was performed 10 times. 


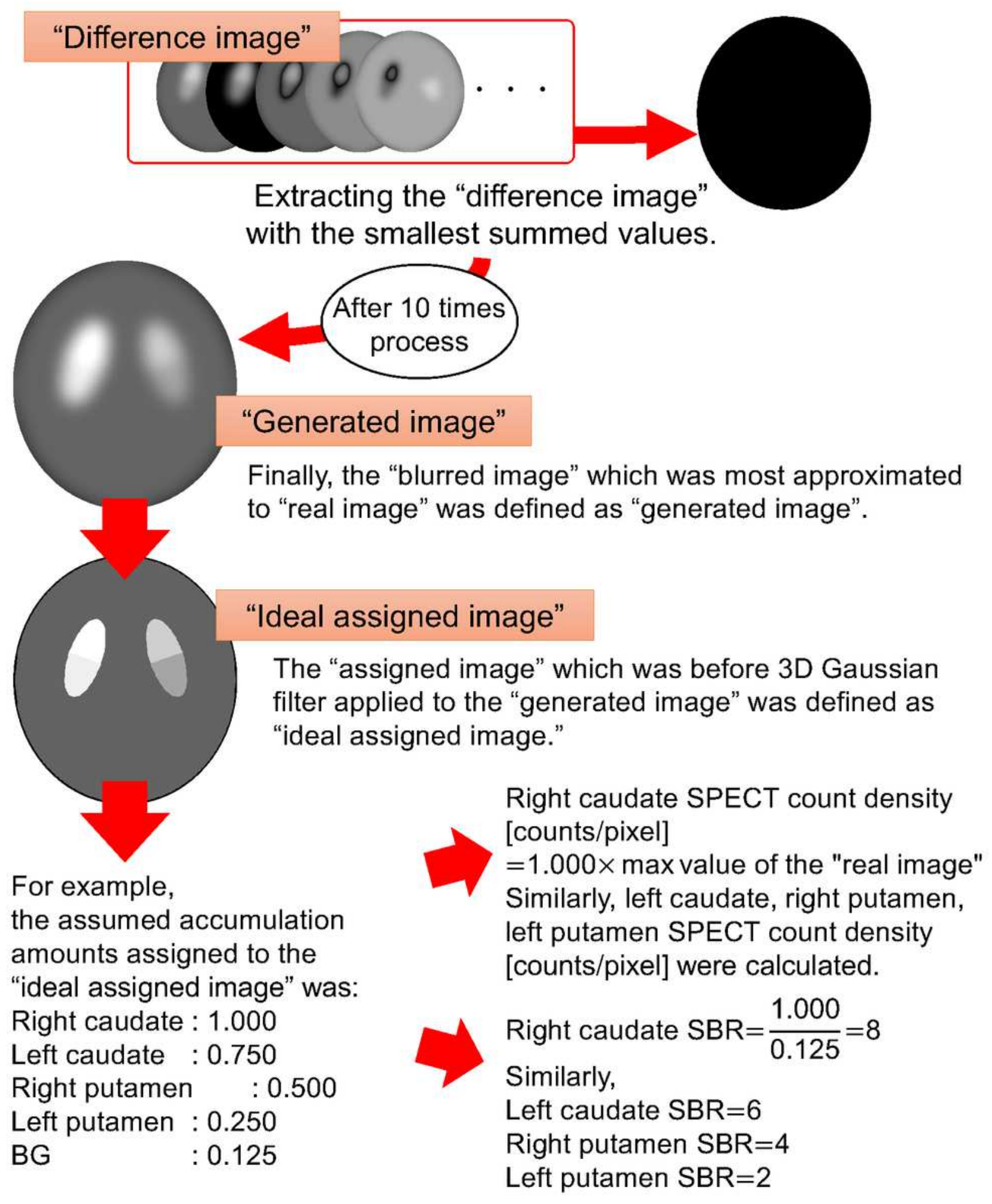

Figure 7

Process No. 6 of the proposed method: Determining the final accumulation amounts and calculating indices After performing the series in Process No. 2-No. 510 times, the "blurred image" finally most approximated to the "real image" was defined as the "generated image." The "generated image" was returned to the image which was before the three-dimensional Gaussian filter was applied in Process No. 2. This ideal image with no blurs was defined as the "ideal assigned image." The assumed accumulation 
amounts in the left and right caudate/putamen and background (BG) regions assigned into the "ideal assigned image" were determined as the accumulation amounts in each region. Using these values, the specific binding ratio (SBR) and the caudate-putamen ratio (CPR) were calculated.
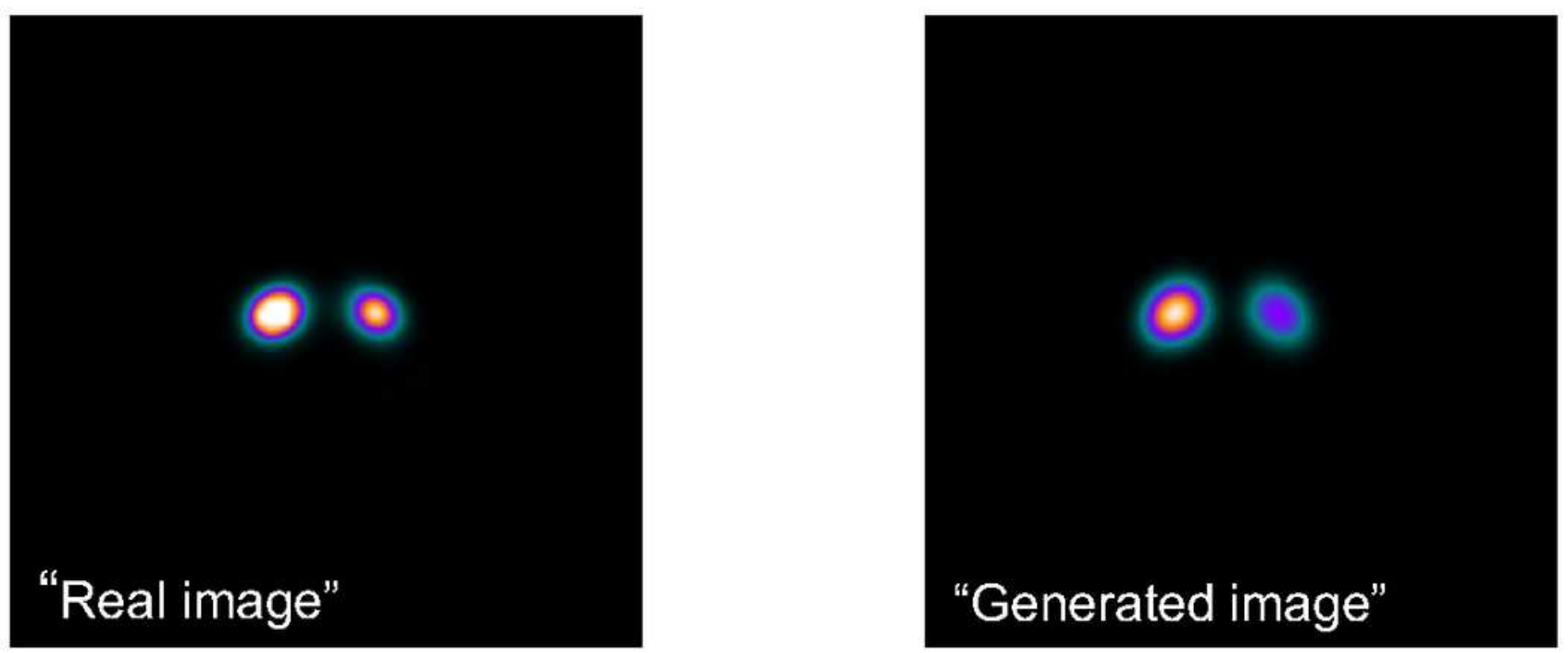

(a)
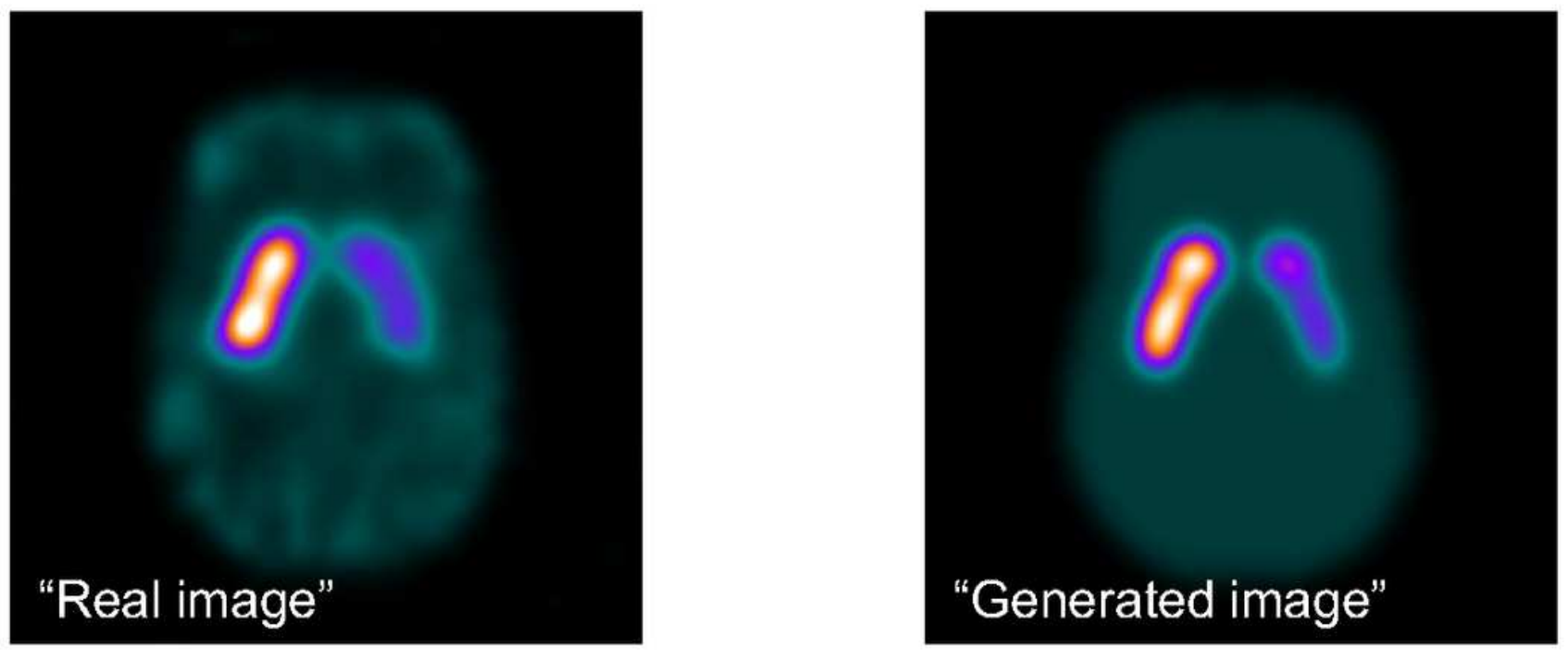

(b)

\section{Figure 8}

Comparison between the "real image" and "generated image" The "Real image" was the image obtained by the actual imaging and the "generated image" was the final image obtained by the proposed method. (a) shows the "real image" and "generated image" obtained from "Phantom1" and (b) shows the image obtained by "Phantom2." These images were in the same display condition for comparison between the 
"real image" and the "generated image." The counts and contrasts of the caudate, putamen, and background $(B G)$ in the "generated image" were visually similar to those in the "real image."
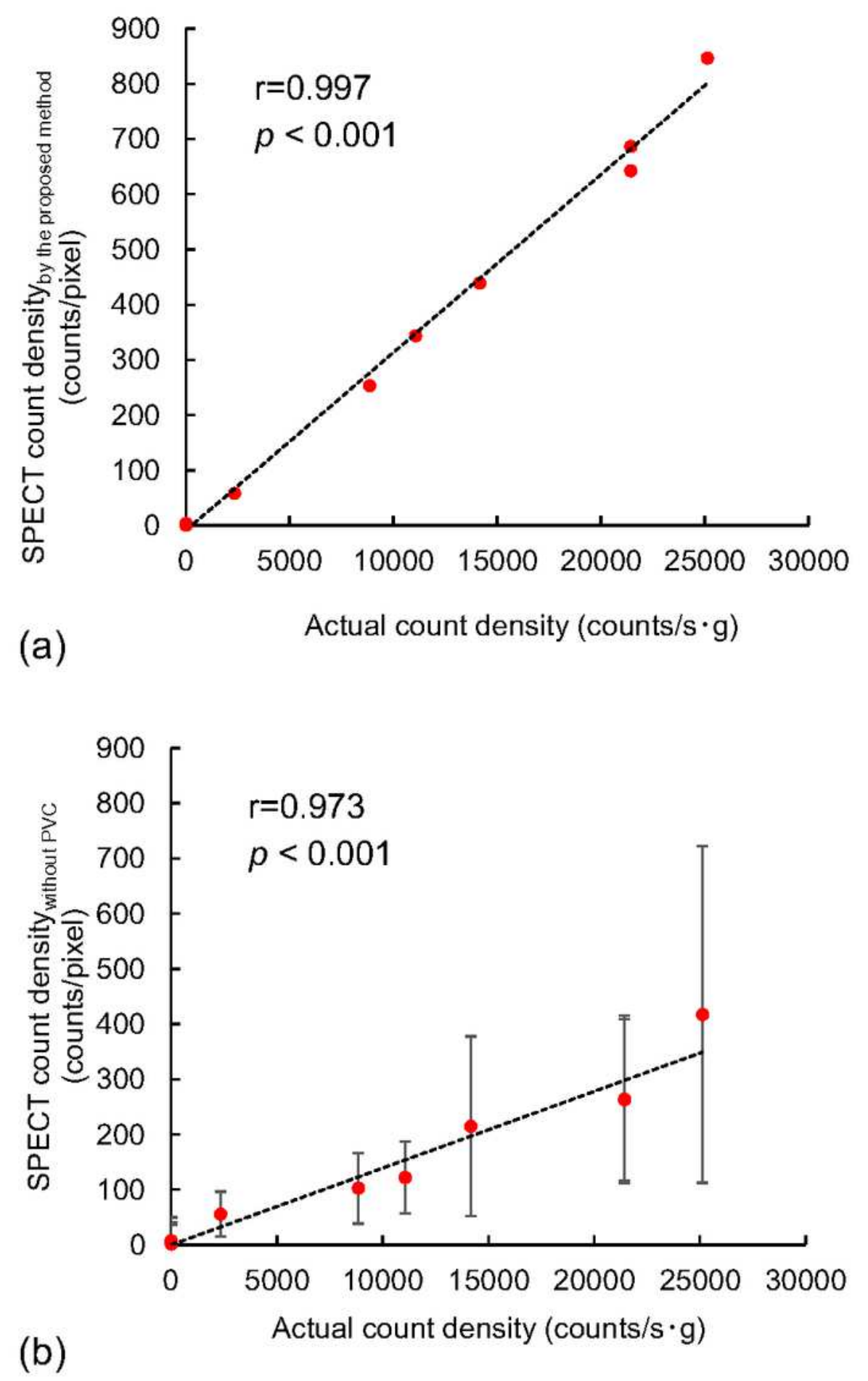

Figure 9

Correlation between the actual count density and the single-photon emission computed tomography (SPECT) count density This figure shows the correlation between the actual count density of the 123I solution filled in the phantom and the SPECT count density. (a) shows the correlation between the actual 
count density and SPECT count densityby the proposed method. The SPECT count densityby the proposed method significantly correlated with the actual count density $(r=0.997, p<0.001)$. (b) shows the correlation between the actual count density and SPECT count densitywithout PVC obtained by the comparison method. The SPECT count densitywithout PVC significantly correlated with the actual count density $(r=0.973, p<0.001)$.

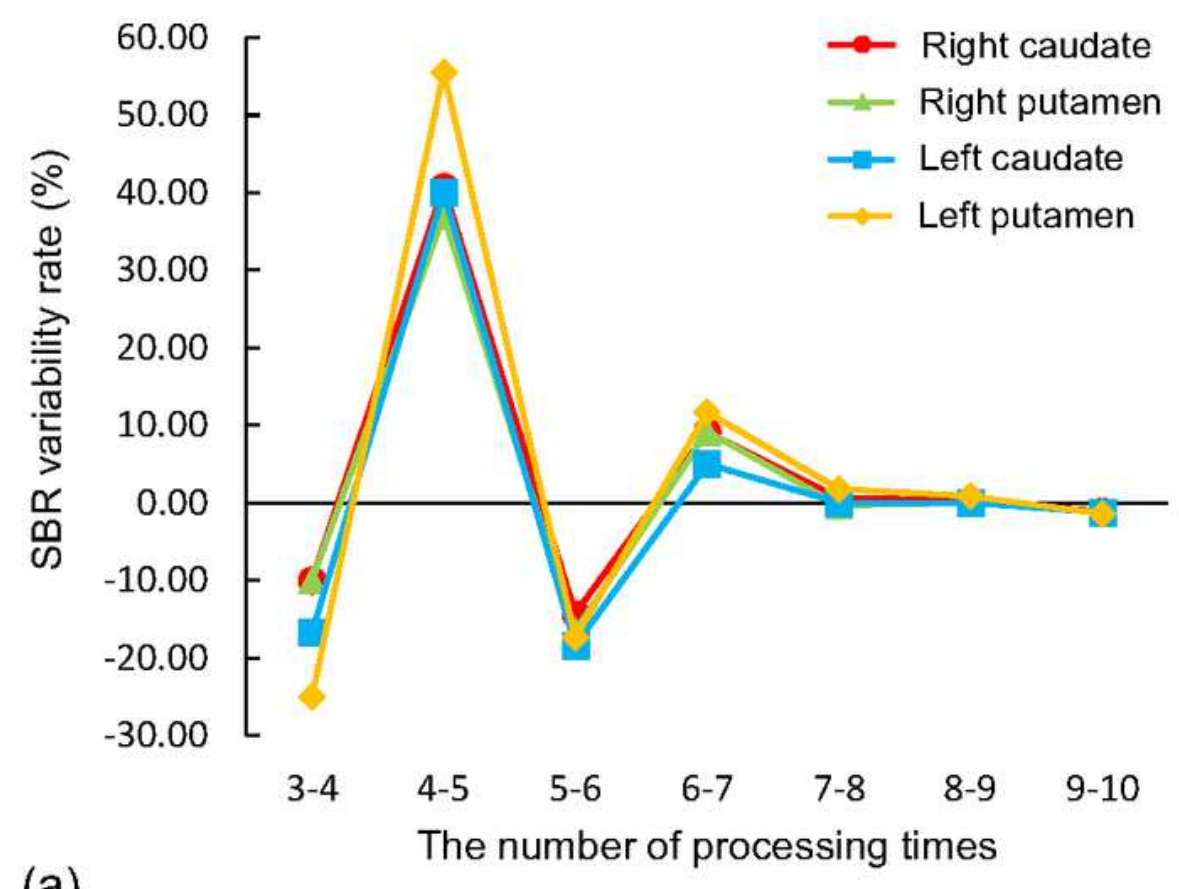

(a)

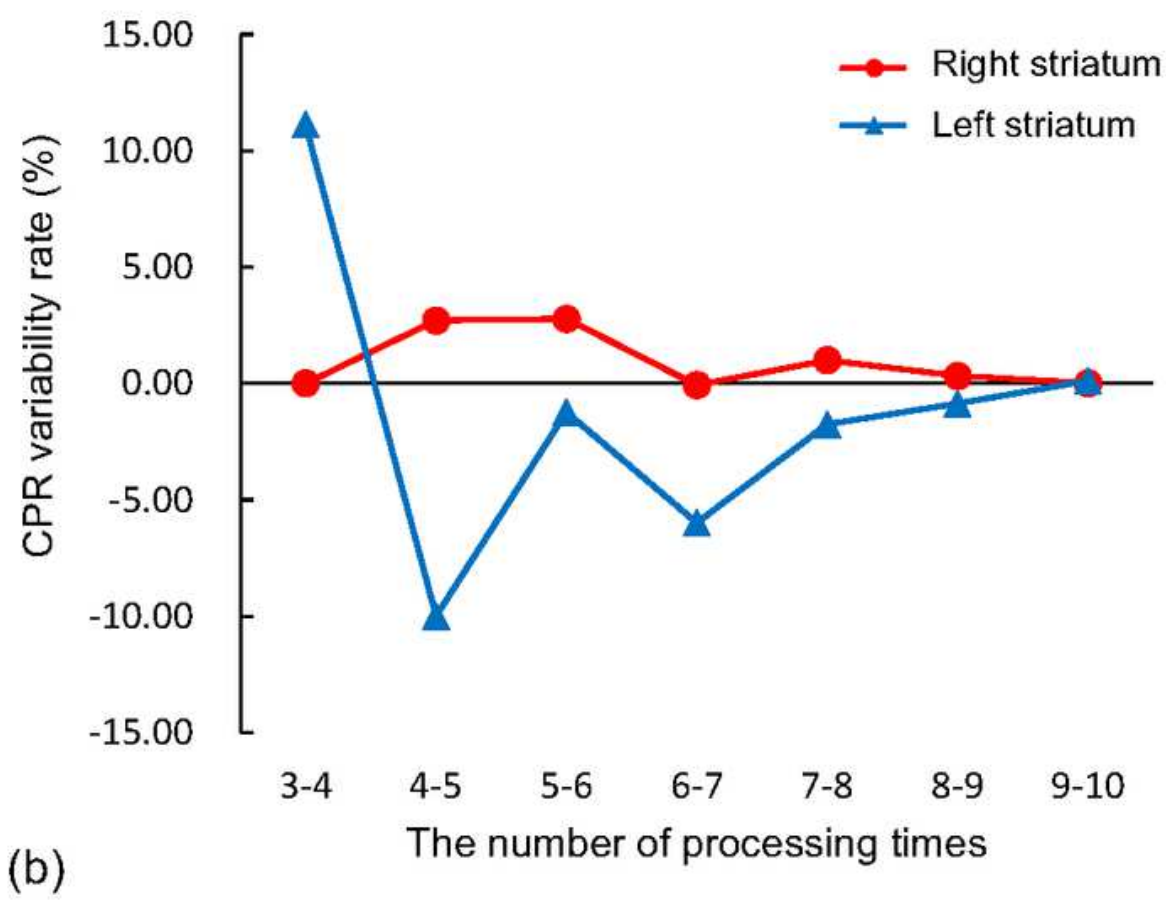

Figure 10 
The relationship between the number of processing times and the variability rate (a) shows the relation between the number of processing times and the variability of specific binding ratio (SBR). When the number of processing times was low, SBRs were greatly variable. However, SBRs were converged as the processing was repeated. When the number of processing times was more than seven, the variability rates of the SBR were less than $5 \%$. (b) shows the relation between the number of processing times and the variability of caudate/putamen ratio (CPR). This relation showed a similar trend to the SBR. 